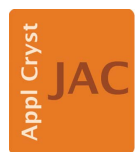

JOURNAL OF

APPLIED

CRYSTALLOGRAPHY

ISSN 1600-5767

Received 27 April 2018

Accepted 27 April 2018

Keywords: X-ray solution scattering; proteins; coarse-graining; MARTINI; structural dynamics; small-angle $X$-ray scattering (SAXS); wide-angle $\mathrm{X}$-ray scattering (WAXS); protein structure refinement

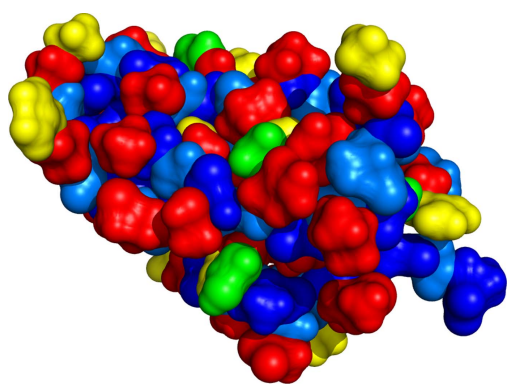

OPEN $\odot$ ACCESS

\section{MARTINI bead form factors for the analysis of time- resolved X-ray scattering of proteins. Erratum}

\author{
Stephan Niebling, Alexander Björling and Sebastian Westenhoff*
}

Department of Chemistry and Molecular Biology, University of Gothenburg, Box 462, SE-40530 Gothenburg, Sweden. *Correspondence e-mail: westenho@chem.gu.se

A typographical error in the article by Niebling, Björling \& Westenhoff [J. Appl. Cryst. (2014), 47, 1190-1198] is corrected.

There is a typographical error in Table 1 of the article by Niebling et al. (2014). The right entry for the second line should be

ARG BB 2211010.69

instead of

ALA BB 3511010.69 .

The authors thank Darren Hsu for pointing out this error.

\section{References}

Niebling, S., Björling, A. \& Westenhoff, S. (2014). J. Appl. Cryst. 47, 1190-1198. 
Journal of

\section{Applied Crystallography}

ISSN 1600-5767

Received 18 December 2013

Accepted 2 May 2014

\section{MARTINI bead form factors for the analysis of time- resolved X-ray scattering of proteins}

\author{
Stephan Niebling, Alexander Björling and Sebastian Westenhoff*
}

Department of Chemistry and Molecular Biology, University of Gothenburg, Box 462, SE-40530 Gothenburg, Sweden. Correspondence e-mail: westenho@chem.gu.se

\begin{abstract}
Time-resolved small- and wide-angle X-ray scattering (SAXS and WAXS) methods probe the structural dynamics of proteins in solution. Although technologically advanced, these methods are in many cases limited by data interpretation. The calculation of X-ray scattering profiles is computationally demanding and poses a bottleneck for all SAXS/WAXS-assisted structural refinement and, in particular, for the analysis of time-resolved data. A way of speeding up these calculations is to represent biomolecules as collections of coarse-grained scatterers. Here, such coarse-graining schemes are presented and discussed and their accuracies examined. It is demonstrated that scattering factors coincident with the popular MARTINI coarse-graining scheme produce reliable difference scattering in the range $0<q<0.75 \AA^{-1}$. The findings are promising for future attempts at X-ray scattering data analysis, and may help to bridge the gap between time-resolved experiments and their interpretation.
\end{abstract}

\section{Introduction}

$\mathrm{X}$-ray solution scattering is a popular technique for gathering structural information on biomolecules in solution (Petoukhov \& Svergun, 2007; Koch et al., 2003; Svergun \& Koch, 2003; Makowski, 2010; Ihee et al., 2010; Westenhoff et al., 2010; Andersson et al., 2009; Cho et al., 2010; Malmerberg et al., 2011; Kim, Muniyappan et al., 2012; Kim, Lee et al., 2012; Ibrahimkutty et al., 2011; Spilotros et al., 2012; Takala et al., 2014). The angular intensity distribution of scattered X-rays is recorded and advanced computational algorithms are available to determine three-dimensional structures from the scattering patterns (Konarev et al., 2006; Petoukhov et al., 2012; Liu et al., 2012). X-ray scattering at small angles (SAXS) provides information on molecular envelopes. At wider angles (WAXS), higher-resolution information is encoded, but low scattering strength and a lack of uniqueness when assigning structural features to the data hinders its practical application.

Time-resolved X-ray solution scattering is an emerging technique for observing structural changes of proteins (Ihee $e t$ al., 2010; Westenhoff et al., 2010; Andersson et al., 2009; Makowski, 2010; Cho et al., 2010; Malmerberg et al., 2011; Kim, Muniyappanet al., 2012; Kim, Lee et al., 2012; Ibrahimkutty et al., 2011; Spilotros et al., 2012; Takala et al., 2014). $\mathrm{X}$-ray scattering is recorded as a function of reaction time and referenced to the scattering patterns of the reactants. The difference technique makes it possible to access higher spatial resolution by detecting WAXS, since all background signals are very precisely canceled. At modern synchrotron facilities the time resolution is limited to approximately $100 \mathrm{ps}$, but free-electron laser sources increase the resolution to $<100 \mathrm{fs}$. This opens up the way for studies of elementary structural changes in proteins on the time scale of atomic motions.
Today the bottleneck in protein solution X-ray scattering lies in interpreting the experimental data. One is forced to model it in an iterative fashion, and to calculate scattering patterns of a large number of trial structures. Since the total scattering is a result of pairwise interference between all the atoms in a protein, each such calculation is time consuming and refinement quickly becomes too computationally demanding.

For a realistic representation of scattering from a molecule in solution, the contributions to the form factor from the electron density of the molecule, the electron density of the displaced solvent and any excess electron density of the solvation shell have to be evaluated. The first term is often computed from the atomic coordinates of the molecule. To represent the solvent displaced by the solute, it is common practice to use modified atomic scattering factors (Fraser et al., 1978). Whereas the use of this approximation is justified at small angles, systematic deviations are introduced for higher angles (Bardhan et al., 2009). The excess electron density in the solvation shell is often modeled as a homogeneous border layer, as implemented in the popular program CRYSOL (Svergun et al., 1995). However this strategy is problematic as two parameters describing the solvation shell are introduced and adjusted ad hoc. Recent developments include explicit solvent treatment (Grishaev et al., 2010; Park et al., 2009) or a more realistic representation of the solute-solvent boundary (Bardhan et al., 2009; Virtanen et al., 2011). By using these more sophisticated methods, the reliability $q$ range can be extended to higher values. However these methods are computationally demanding and it is hard to prove their accuracy experimentally. We show explicitly below that the need for accurate computation of the solvent layer is relaxed when analyzing difference X-ray scattering. 
Given that solution scattering signals of proteins do not encode enough information to reveal atomic level details, a coarse-grained representation should often be suitable for interpreting SAXS and WAXS data. Such representations greatly decrease the computational cost of predicting X-ray scattering curves (Yang et al., 2009; Stovgaard et al., 2010; Zheng \& Tekpinar, 2011; Daily et al., 2012), rendering ambitious iterative refinement schemes realistic for relatively large protein systems. In recent years, the MARTINI model, based on coarse-grained representations of biomolecules, has become popular for simulating the dynamics of various biological systems (Marrink et al., 2007; Monticelli et al., 2008; López et al., 2009; Yesylevskyy et al., 2010; de Jong et al., 2013; Marrink \& Tieleman, 2013). It drastically reduces the computational cost of molecular dynamics simulations, allowing simulation on longer time scales and of larger systems with modest computational resources. The force field is designed to reproduce thermodynamic data and has been successfully applied to several simulation problems, for example, protein-lipid interactions (van den Bogaart et al., 2011; Schäfer et al., 2011; Louhivuori et al., 2010).

In this study, we describe and compare methods for coarsegrained X-ray scattering calculations, especially aiming at the analysis of time-resolved difference scattering. We show how difference X-ray scattering profiles can be calculated efficiently from MARTINI coarse-grained representations of proteins and we assess the reliability limits for these calculations. We find that coarse-grained scattering calculations are reliable in a larger $q$ range for difference scattering compared with absolute scattering. We conclude that this method opens up a way for structural refinement routines of large proteins, especially in combination with time-resolved SAXS/WAXS experiments.

\section{Theory and methods}

\subsection{X-ray scattering from coarse-grained structures}

The scattering amplitude from a collection of point-like atomic scatterers (Warren, 1990) is described by

$$
F(\mathbf{q})=\sum_{k} f_{k} \exp \left(i \mathbf{q} \cdot \mathbf{r}_{k}\right),
$$

where $\mathbf{q}$ is the scattering vector and $\mathbf{r}_{k}$ and $f_{k}$ are the position and the scattering factor of atom $k$, respectively. For randomly oriented molecules in solution, the scattered intensity is obtained by multiplying this sum by its complex conjugate,

$$
\begin{aligned}
I(\mathbf{q}) & =\left[\sum_{k} f_{k} \exp \left(i \mathbf{q} \cdot \mathbf{r}_{k}\right)\right]\left[\sum_{l} f_{l} \exp \left(-i \mathbf{q} \cdot \mathbf{r}_{l}\right)\right] \\
& =\sum_{k} \sum_{l} f_{k} f_{l} \exp \left(i \mathbf{q} \cdot \mathbf{r}_{k l}\right),
\end{aligned}
$$

and then taking the spherical average with $q=|\mathbf{q}|=$ $4 \pi(\sin \theta / \lambda)$, where $\lambda$ is the wavelength of the radiation, $2 \theta$ is the scattering angle and $\mathbf{r}_{k l}$ is the vector from scatterer $l$ to scatterer $k$. Equation (2) becomes

$$
\begin{aligned}
I(q) & =\langle I(\mathbf{q})\rangle=\left\langle\sum_{k} \sum_{l} f_{k} f_{l} \exp \left(i \mathbf{q} \cdot \mathbf{r}_{k l}\right)\right\rangle \\
& =\sum_{k} \sum_{l} f_{k} f_{l}\left\langle\exp \left(i \mathbf{q} \cdot \mathbf{r}_{k l}\right)\right\rangle=\sum_{k} \sum_{l} f_{k} f_{l} \frac{\sin \left(q r_{k l}\right)}{q r_{k l}} .
\end{aligned}
$$

The last result is known as the Debye equation and can be used to predict the vacuum scattering of a biomolecule.

A complication is that solution scattering patterns contain a large undesired solvent signal. This signal can be removed by subtracting a buffer background, but at the cost of including a negative term for the displaced solvent that must be accounted for in predicted data. It can be introduced in an approximate way, at the level of the atomic scattering factors, so that the unmodified Debye equation [equation (3)] can still be used. Such corrected atomic scattering factors $f_{k}^{\text {excl }}(q)$ are derived from $f_{k}(q)$ by subtracting a Gaussian sphere representing the scattering amplitude of the displaced solvent (Fraser et al., 1978):

$$
f_{k}^{\mathrm{excl}}(q)=f_{k}(q)-v_{k} \rho_{\mathrm{s}} \exp \left(-\pi v_{k}^{2 / 3} q^{2}\right) .
$$

Here $v_{k}$ is the tabulated (Fraser et al., 1978) volume for each atom and $\rho_{\mathrm{s}}$ is the mean electron density of the bulk solvent. In the remainder of this paper, all scattering factors contain this displaced-solvent term unless stated otherwise.

For biomolecules, the computational cost for evaluating equation (3) can be quite high. This is especially important for iterative structural refinement procedures where many test structures have to be evaluated. One strategy for decreasing computational cost is to use a coarse-grained representation of the structure, where each coarse bead represents a group of atoms. It is convenient if each bead is described by a scattering factor $F(q)$, so that the scattering intensity is given by a coarsegrained Debye equation, where the indices $(m, n)$ denote coarse beads and $R_{m n}$ the distance between them:

$$
I(q)=\sum_{m} \sum_{n} F_{m}(q) F_{n}(q) \frac{\sin \left(q R_{m n}\right)}{q R_{m n}} .
$$

Finding these $F_{m}(q)$ is not trivial and we review some possibilities before describing the approach taken in this study.

2.1.1. The bead position approximation. The simplest way to express the overall scattering in terms of coarse-grained scattering factors is to consider each atom to be located at the center of its bead. All atom-atom distances are then approximated by the corresponding bead-bead distances. Where $(k, l)$ are atomic indices and $(m, n)$ denote coarse beads, the Debye equation can be written as follows:

$$
\begin{aligned}
I(q) & =\sum_{k} \sum_{l} f_{k} f_{l} \frac{\sin \left(q r_{k l}\right)}{q r_{k l}} \\
& =\sum_{m} \sum_{n} \sum_{k \in m} \sum_{l \in n} f_{k} f_{l} \frac{\sin \left(q r_{k l}\right)}{q r_{k l}} \\
& \simeq \sum_{m} \sum_{n} \sum_{k \in m} \sum_{l \in n} f_{k} f_{l} \frac{\sin \left(q R_{m n}\right)}{q R_{m n}} \\
& =\sum_{m} \sum_{n} \frac{\sin \left(q R_{m n}\right)}{q R_{m n}}\left(\sum_{k \in m} f_{k}\right)\left(\sum_{l \in n} f_{l}\right) .
\end{aligned}
$$


We then have

$$
F_{m} \simeq \sum_{k \in m} f_{k} .
$$

In this approximation, the internal structure of the beads is completely ignored. We note that equation (7) holds exactly for $q=0$.

2.1.2. The spherical 'glob' approximation. Another option is to take, for each bead, the spherical average of the amplitude [equation (1)] before taking intensities. This is equivalent to smearing each atom out on a sphere of radius $r_{k}$ around the center of the bead (Harker, 1953):

$$
\begin{aligned}
F_{n}(q) & =\left\langle F_{n}(\mathbf{q})\right\rangle=\left\langle\sum_{k \in n} f_{k} \exp \left(i \mathbf{q} \cdot \mathbf{r}_{k}\right)\right\rangle \\
& =\sum_{k \in n} f_{k} \frac{\sin \left(q r_{k}\right)}{q r_{k}} .
\end{aligned}
$$

Here, the distance of each atom to the center of the beads is considered, but the angular arrangement of the atoms is ignored.

2.1.3. The self-consistent set approximation. The most general approach described in this paper is to find, for a given set of proteins, a self-consistent set of $F(q)$ values that reproduces all pairwise bead-bead scattering intensity terms of the corresponding atomistic structures as well as possible. Considering two beads with scattering factors $F_{\mathrm{A}}$ and $F_{\mathrm{B}}$, the total scattering intensity of the pair $I_{\mathrm{AB}}$ is given by equation (3). These quantities are related by applying equation (5) to the pair of beads:

$$
I_{\mathrm{AB}}=F_{\mathrm{A}}^{2}+F_{\mathrm{B}}^{2}+2 F_{\mathrm{A}} F_{\mathrm{B}} \frac{\sin \left(q R_{\mathrm{AB}}\right)}{q R_{\mathrm{AB}}} .
$$

If $F_{\mathrm{B}}$ is held constant in the comparison, $F_{\mathrm{A}}$ is obtained by solving this quadratic equation, choosing the correct root by comparing to equation (7), which actually holds for $q=0$. A self-consistent set of coarse-grained scattering factors can be found from the following scheme.

(a) Generate starting guesses for the bead form factors, for example, by using equations (7) or (8).

(b) Pick a random bead in the structure and call it A.

(c) Go through all other beads in the structure, letting each act as B, and calculate $F_{\mathrm{A}}$ for each case according to equation (9).

(d) Take the average of all these $F_{\mathrm{A}}$, and assign it to bead A.

(e) Repeat $(b)-(d)$ until the set form factors have converged.

2.1.4. The single-bead approximation. Although conceptually simple, the last approach is cumbersome, especially for large libraries of proteins. Yang et al. (2009) have presented a simpler approach, where form factors are chosen such that they reproduce the scattering intensities of isolated beads. In this approach, numerically correct coarse-grained form factors for entire amino acid residues can be obtained simply by taking the square root of the scattering intensity from a group of atoms:

$$
F_{m}(q)=\left[\sum_{k \in m} \sum_{l \in m} f_{k} f_{l} \frac{\sin \left(q r_{k l}\right)}{q r_{k l}}\right]^{1 / 2} .
$$

We note that this equation can only produce positive form factors, which is not correct in general when the negative term for the displaced solvent is included. For $q=0$, the value of the form factor of the bead must equal the sum of the atomic scattering factors:

$$
F_{m}(q=0)=\sum_{k \in m} f_{k}(q=0) .
$$

With water as a solvent, $f_{k}(q=0)$ is negative for the hydrogen atom when the displaced-solvent contribution is included. Thus, negative values of $F(q)$ can occur for beads containing hydrogen atoms $[f(q=0)=-0.72$ electron units (e.u.)]. Therefore, form factors obtained using equation (10) that do not satisfy equation (11) must be corrected. In practice, this is the case for side-chain beads which contain only hydrogen and carbon atoms. When the beads consist of entire amino acids the $f(q=0)$ values are generally positive (Yang et $a l ., 2009)$. To correct the scattering factors that do not satisfy equation (11), we use a common feature of all these scattering factors, which is the appearance of a minimum with two associated inflection points (Fig. 1). The data with $q$ larger than the high- $q$ inflection point are used for a sixth-order polynomial fit, constrained at $q=0$ to satisfy equation (11). This polynomial is then accepted as the actual form factor of the coarse bead (Fig. 1).

To illustrate the differences between the above four approaches, the coarse-grained X-ray scattering of hen eggwhite lysozyme was calculated from a MARTINI (the MARTINI coarse-grained representation of proteins will be described later in \$2.2) coarse-grained structure and compared with the all-atom calculation (Fig. 2). To enable a direct comparison of the form factor calculation methods, the scat-

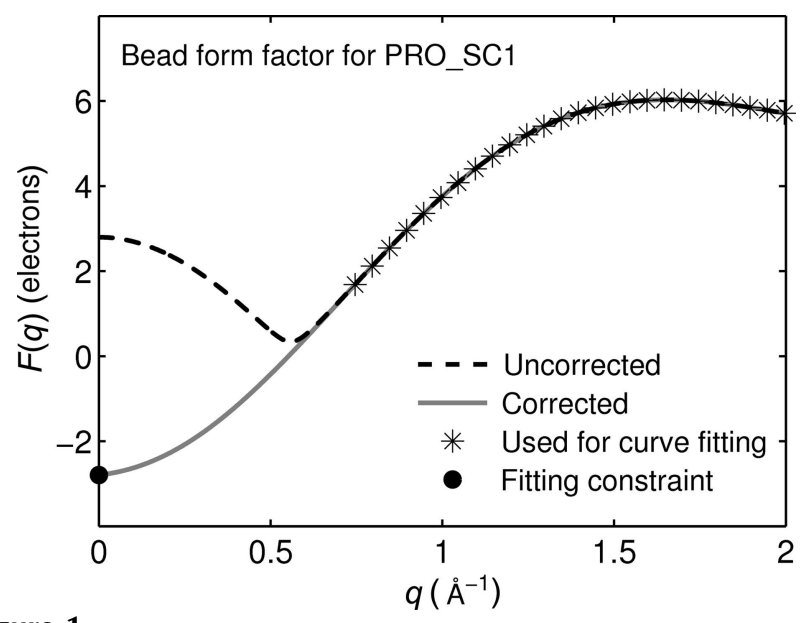

Figure 1

Example of bead scattering factor correction (proline, side chain 1): the dashed curve is calculated according to equation (10). The value for $q=0$ does not satisfy equation (11) ( $c f$. Table 1 ). Therefore only the points after the inflection point (following the minimum) were used for the sixth-order polynomial curve fitting (stars). The value at $q=0$ (from Table 1 ) is used as an equality constraint (filled circle). This yields the corrected bead scattering factor (solid line). 
tering was calculated without the displaced-solvent model. For $q<0.25 \AA^{-1}$, all approaches are in good agreement with the all-atom calculation. This is reasonable because long interbead distances are probed in this $q$ range. Regarding the high$q$ region, the bead-position approximation shows significant deviations for $q>0.4 \AA^{-1}$. The spherical glob approximation reproduces the all-atom calculation well for $q<1.2 \AA^{-1}$ but deviates for larger $q$ values. The self-consistent set approximation and the single-bead approximation yield almost identical results and agree relatively well with the all-atom calculations even for high $q$ values.

This degree of agreement corresponds to the order with which the internal structure of the bead is accounted for: the bead-position approximation neglects the internal structure, the spherical glob approximation smears the atoms out on spheres around its center, and the single-bead approximation as well as the self-consistent set approximation include the internal bead structure most accurately. The latter two approximations agree remarkably well with the all-atom calculation. The single-bead approximation is computationally less expensive than the self-consistent set approximation. We therefore chose to use the former for calculating coarsegrained scattering factors in the remainder of this study.

\subsection{Application to the MARTINI model}

We now turn our attention to applying the single-bead approximation to the MARTINI model (Marrink et al., 2007; de Jong et al., 2013; Marrink \& Tieleman, 2013) as an efficient way to calculate X-ray scattering from coarse-grained structures. In the MARTINI model, four non-hydrogen atoms and their associated hydrogen atoms are mapped, on average, onto one bead, with each amino acid residue composed of a backbone bead and up to four side-chain beads (Monticelli et al., 2008). The beads are grouped by their polarity and hydrogen bonding ability, yielding a total of 20 different bead types whose interactions are specified by the MARTINI force field.

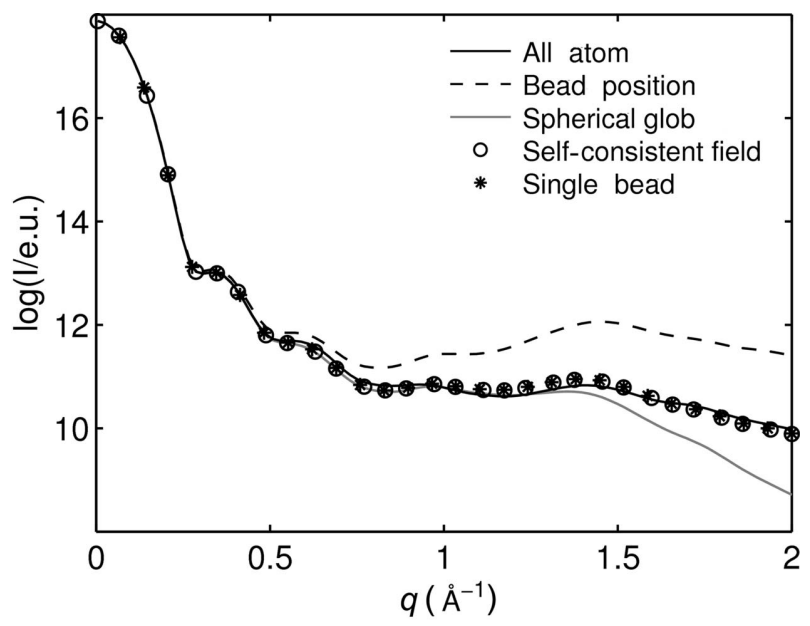

Figure 2

Comparison of the four ways of determining coarse-grained form factors. All calculations were performed without the displaced-solvent model for hen egg-white lysozyme (PDB code 6lyz).
Table 1

Bead types used for the coarse-grained X-ray scattering calculations and their elemental formulae.

The sums of the atomic scattering factors at $q=0$, corrected with the displaced-solvent model, are shown in the last columns. The form factors of all beads with $\sum f(q=0)<0$, marked in bold, were corrected as described in the text.

\begin{tabular}{|c|c|c|c|c|c|c|c|}
\hline \multirow[b]{2}{*}{ AA } & \multirow[b]{2}{*}{ Bead } & \multicolumn{5}{|c|}{ Number of atoms } & \multirow[b]{2}{*}{$f(q=0$} \\
\hline & & $\mathrm{C}$ & $\mathrm{H}$ & $\mathrm{N}$ & $\mathrm{O}$ & $\mathrm{S}$ & \\
\hline ALA & BB & 3 & 5 & 1 & 1 & 0 & 9.04 \\
\hline ALA & BB & 3 & 5 & 1 & 1 & 0 & 10.69 \\
\hline ARG & SC1 & 3 & 6 & 0 & 0 & 0 & -2.79 \\
\hline ARG & $\mathrm{SC} 2$ & 1 & 5 & 3 & 0 & 0 & 15.39 \\
\hline ASN & BB & 2 & 2 & 1 & 1 & 0 & 10.69 \\
\hline ASN & SC1 & 2 & 4 & 1 & 1 & 0 & 9.25 \\
\hline ASP & BB & 2 & 2 & 1 & 1 & 0 & 10.69 \\
\hline ASP & $\mathrm{SC} 1$ & 2 & 2 & 0 & 2 & 0 & 9.48 \\
\hline CYS & BB & 2 & 2 & 1 & 1 & 0 & 10.69 \\
\hline CYS & $\mathrm{SC} 1$ & 1 & 2 & 0 & 0 & 1 & 8.44 \\
\hline GLN & BB & 2 & 2 & 1 & 1 & 0 & 10.69 \\
\hline GLN & SC1 & 3 & 6 & 1 & 1 & 0 & 8.32 \\
\hline GLU & BB & 2 & 2 & 1 & 1 & 0 & 10.69 \\
\hline GLU & $\mathrm{SC} 1$ & 3 & 4 & 0 & 2 & 0 & 8.55 \\
\hline GLY & BB & 2 & 3 & 1 & 1 & 0 & 9.97 \\
\hline HIS & BB & 2 & 2 & 1 & 1 & 0 & 10.69 \\
\hline HIS & SC1 & 2 & 2 & 0 & 0 & 0 & -0.42 \\
\hline HIS & $\mathrm{SC} 2$ & 1 & 1 & 1 & 0 & 0 & 5.95 \\
\hline HIS & SC3 & 1 & 1 & 1 & 0 & 0 & 5.95 \\
\hline ILE & BB & 2 & 2 & 1 & 1 & 0 & 10.69 \\
\hline ILE & SC1 & 4 & 9 & O & 0 & 0 & -4.44 \\
\hline LEU & BB & 2 & 2 & 1 & 1 & 0 & 10.69 \\
\hline LEU & SC1 & 4 & 9 & 0 & 0 & 0 & -4.44 \\
\hline LYS & BB & 2 & 2 & 1 & 1 & 0 & 10.69 \\
\hline LYS & SC1 & 3 & 6 & 0 & 0 & 0 & -2.79 \\
\hline LYS & $\mathrm{SC} 2$ & 1 & 5 & 1 & 0 & 0 & 3.07 \\
\hline MET & BB & 2 & 2 & 1 & 1 & 0 & 10.69 \\
\hline MET & SC1 & 3 & 7 & 0 & 0 & 1 & 5.86 \\
\hline PHE & BB & 2 & 2 & 1 & 1 & 0 & 10.69 \\
\hline PHE & SC1 & 3 & 3 & $\mathbf{0}$ & $\mathbf{0}$ & $\mathbf{0}$ & -0.63 \\
\hline PHE & SC2 & 2 & 2 & o & o & o & -0.42 \\
\hline PHE & SC3 & 2 & 2 & 0 & 0 & 0 & -0.42 \\
\hline PRO & BB & 2 & 1 & 1 & 1 & 0 & 11.41 \\
\hline PRO & SC1 & 3 & 6 & O & 0 & o & -2.79 \\
\hline SER & BB & 2 & 2 & 1 & 1 & 0 & 10.69 \\
\hline SER & SC1 & 1 & 3 & 0 & 1 & 0 & 3.30 \\
\hline THR & BB & 2 & 2 & 1 & 1 & 0 & 10.69 \\
\hline THR & $\mathrm{SC} 1$ & 2 & 5 & 0 & 1 & 0 & 2.37 \\
\hline TRP & BB & 2 & 2 & 1 & 1 & 0 & 10.69 \\
\hline TRP & SC1 & 3 & 2 & 0 & 0 & 0 & 0.09 \\
\hline TRP & $\mathrm{SC} 2$ & 2 & 2 & 1 & 0 & 0 & 5.74 \\
\hline TRP & SC3 & 2 & 2 & 0 & 0 & 0 & -0.42 \\
\hline TRP & SC4 & 2 & 2 & 0 & 0 & 0 & -0.42 \\
\hline TYR & BB & 2 & 2 & 1 & 1 & 0 & 10.69 \\
\hline TYR & SC1 & 3 & 3 & O & 0 & o & -0.63 \\
\hline TYR & SC2 & 2 & 2 & 0 & 0 & 0 & -0.42 \\
\hline TYR & SC3 & 2 & 2 & 0 & 1 & 0 & 4.53 \\
\hline VAL & BB & 2 & 2 & 1 & 1 & 0 & 10.69 \\
\hline VAL & SC1 & 3 & 7 & O & 0 & 0 & -3.51 \\
\hline
\end{tabular}

For X-ray scattering calculations the geometrical similarity and the molecular formula (number of electrons) of the beads is of main importance, not the polarity or hydrogen bonding ability. We therefore derive X-ray form factors for each MARTINI bead as it appears in every amino acid residue type. This yields the 49 different scattering types listed in Table 1. The original mapping of atoms into beads according to the MARTINI model is retained for the X-ray scattering calculations. This means that MARTINI coarse-grained structures can be used directly as an input for these calculations. 
Table 2

PDB structures used for the determination of the average bead form factors.

\begin{tabular}{|c|c|c|c|}
\hline PDB entry & Atoms & Amino acids & MARTINI beads \\
\hline 1ARV & 4845 & 336 & 682 \\
\hline 1AVD & 3854 & 247 & 555 \\
\hline 1BP2 & 1842 & 123 & 276 \\
\hline 1BPI & 892 & 58 & 130 \\
\hline $1 \mathrm{COL}$ & 6006 & 394 & 804 \\
\hline $1 \mathrm{CSE}$ & 4827 & 337 & 685 \\
\hline $1 \mathrm{CSH}$ & 6765 & 435 & 952 \\
\hline 1DHR & 3527 & 236 & 493 \\
\hline 1GAL & 8700 & 581 & 1242 \\
\hline $1 \mathrm{HCB}$ & 3973 & 258 & 585 \\
\hline 1HEL & 1960 & 129 & 283 \\
\hline 1HML & 1946 & 123 & 277 \\
\hline 1HRC & 1672 & 104 & 236 \\
\hline 1HRS & 2775 & 174 & 385 \\
\hline 1ISC & 5852 & 384 & 862 \\
\hline $1 \mathrm{LPE}$ & 2364 & 144 & 310 \\
\hline $1 \mathrm{MOL}$ & 3124 & 188 & 440 \\
\hline 1PNK & 11708 & 750 & 1675 \\
\hline $1 \mathrm{PPN}$ & 3245 & 212 & 469 \\
\hline 1RTP & 4974 & 327 & 705 \\
\hline 1SCS & 3564 & 237 & 518 \\
\hline 1SXC & 4320 & 302 & 616 \\
\hline 1THW & 3031 & 207 & 445 \\
\hline $1 \mathrm{TOP}$ & 2466 & 162 & 338 \\
\hline 1UBI & 1231 & 76 & 163 \\
\hline 1XYP & 5636 & 378 & 856 \\
\hline 1YMB & 2411 & 153 & 343 \\
\hline 2AAI & 8212 & 529 & 1149 \\
\hline 2CGA & 7154 & 490 & 1022 \\
\hline 2GST & 7246 & 434 & 1036 \\
\hline $2 \mathrm{OHX}$ & 11278 & 748 & 1576 \\
\hline 2PSG & 5425 & 369 & 780 \\
\hline 2SBL & 25698 & 1614 & 3636 \\
\hline 2ST1 & 3837 & 275 & 540 \\
\hline 2TGA & 3222 & 223 & 465 \\
\hline 3EBX & 920 & 62 & 139 \\
\hline 3PGK & 6376 & 415 & 878 \\
\hline 3PTE & 5163 & 347 & 728 \\
\hline 4CMS & 4854 & 320 & 704 \\
\hline 4PEP & 4672 & 325 & 679 \\
\hline 6RAT & 1857 & 124 & 273 \\
\hline 7TIM & 7556 & 494 & 1050 \\
\hline
\end{tabular}

The average form factors for the MARTINI beads following the single-bead approximation were acquired from a rationally selected library of protein structures that covers a wide range of protein folds and different secondary structure contents (Oberg et al., 2003). We excluded seven proteins with missing non-hydrogen atoms. This resulted in a library of 43 proteins shown in Table 2. Missing hydrogen atoms were added with the pdb2gmx tool which is part of the GROMACS suite (Hess et al., 2008). To investigate the effect of the bead size on accuracy, an additional coarse-grained mapping with one amino acid per bead was used (Yang et al., 2009; Zheng \& Tekpinar, 2011). Both the amino acid and the MARTINI beads were positioned at the centers of mass of the respective atom groups.

To keep these calculations simple and universally applicable a few structural details were ignored. First of all, the N- and Ctermini were not differentiated for the coarse-grained calculation. The respective amino acids were included in the calculation of the average bead scattering factors, and thus the larger number of electrons for the C-terminus is reflected by this averaging. Charged atoms were also ignored, both to limit the number of bead types and since information on the charge is not always available.

\section{Results}

\subsection{The library average of bead form factors}

A central aim of this work is to estimate the reliability of coarse-grained X-ray scattering calculations with respect to the size of the beads. Two coarse-grained mapping schemes were used: the amino acid mapping as used by Yang et al. (2009) and our MARTINI-bead approach. A first comparison can be made at the stage of form factor averaging. The smaller the variation between the individual form factors being averaged, the greater the reliability of the coarse-grained scattering calculation. This is especially important for small proteins or proteins of unusual structure or amino acid content.

The bead scattering factors for all methionines in the library are shown in Fig. 3. The data were computed with equation (10). Deviations for the amino acid bead and the backbone MARTINI bead at $q=0$ are the result of the inclusion of Nterminal amino acids, which contain two additional hydrogen atoms. Since the hydrogen scattering with the displacedsolvent correction is negative for low $q$, the corresponding bead scattering factors for low $q$ are below the majority of the curves.

The calculated form factors for the amino acid beads show a large variation (Fig. $3 a$ ), whereas the form factors of the finer MARTINI beads are more homogeneous (Figs. $3 b$ and $3 c$ ). When the two MARTINI beads (backbone and side chain) are compared, the backbone bead scattering factors are more heterogeneous, whereas the side-chain scattering factors are remarkably well represented by the mean value.

To identify the structural origin of the variation in bead scattering factors, we clustered the protein library based on their prevailing secondary structure. According to the classification of Oberg et al. (2003), the scattering factors of the proteins that show a high percentage of $\alpha$-helices and $\beta$-sheets are shown in different colors (Fig. 3). The MARTINI backbone bead scattering factors clearly cluster into two groups according to secondary structures. In contrast, the amino acid beads do not show such a clear picture.

\subsection{Calculation of difference scattering from coarse-grained protein structures}

The analysis of time-resolved WAXS experiments often requires repeated evaluation of difference scattering from many different trial structures and thus depends on fast but reliable scattering calculations over a $q$ range up to approximately $1 \AA^{-1}$. In order to estimate the accuracy of difference scattering calculations from coarse-grained structures, the predicted difference scattering between the crystal structures of human deoxy hemoglobin (PDB code 2hhb; Fermi et al., 1984) compared with human carbonmonoxy hemoglobin 
(PDB code 1bbb; Silva et al., 1992), as calculated with all-atom and coarse-grained methods, are shown in Fig. 4. This model system has already been used for time-dependent X-ray scattering studies and high-quality data are available (Cammarata et al., 2008). The amino-acid-based result deviates considerably from the all-atom calculation for $q>$ $0.4 \AA^{-1}$, but the MARTINI coarse-grained calculation is accurate for $q<0.75 \AA^{-1}$. This finding is reasonable considering that the level of structural detail is highest in the allatom representation, reduced in the MARTINI coarse-

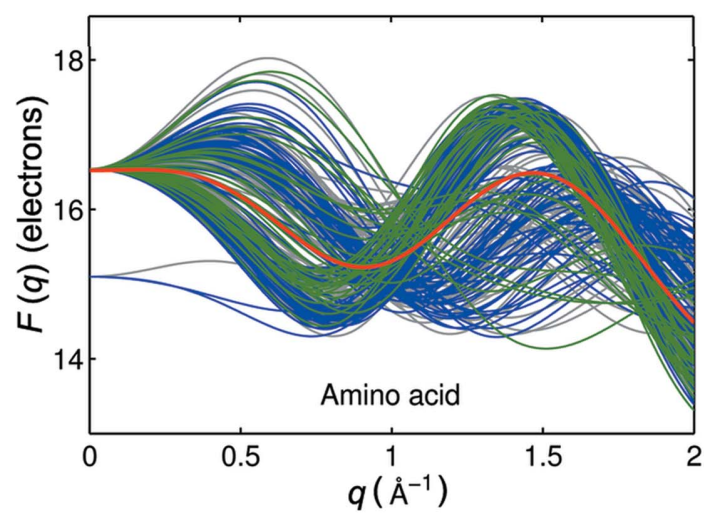

(a)

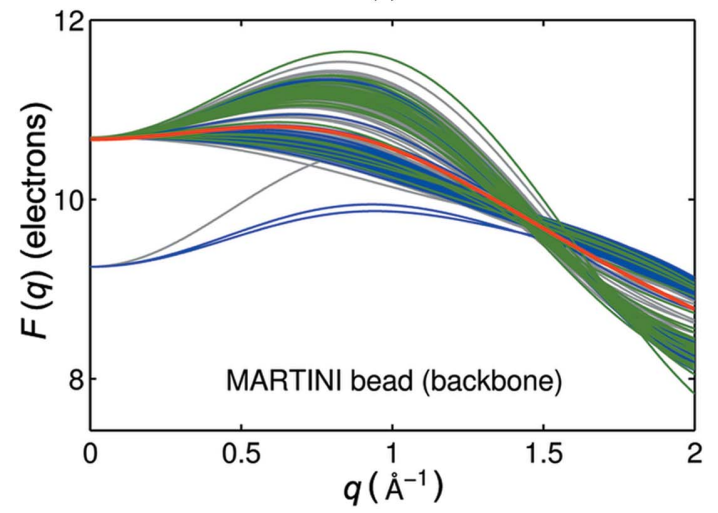

(b)

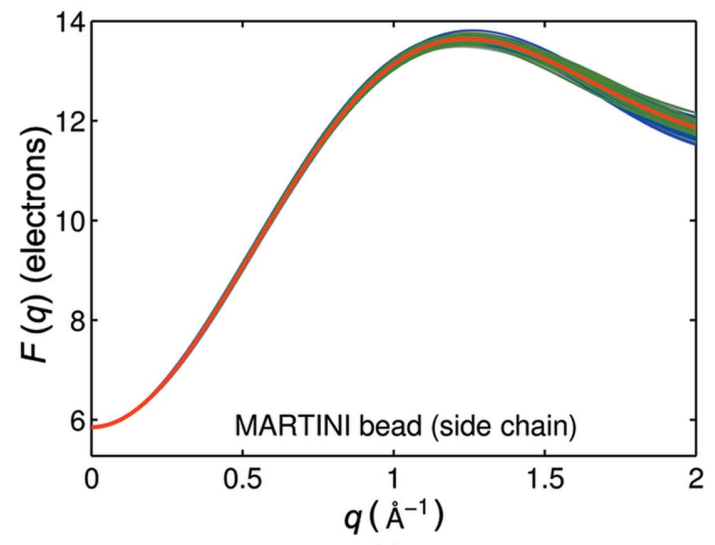

(c)

\section{Figure 3}

Form factors calculated with displaced solvent for all methionines in the library with amino acid $(a)$ and MARTINI bead $(b),(c)$ coarse-graining. Bead form factors are colored according to the prevalent protein secondary structure motif (blue: $\alpha$-helical proteins; green: $\beta$-sheet proteins; gray: unique assignment not possible). The red curves represent the average over all individual form factors. graining scheme and lowest in the amino acid approach. The computation times for the three curves in general scale approximately as 50 (all atom):1 (MARTINI):0.2 (amino acid approach).

Comparing the model calculations with the experimental difference scattering from Cammarata et al. (2008) shows that the agreement between the structural model and the experiment is excellent for $q<0.4 \AA^{-1}$, but that the model fails for higher $q$ values. This is most likely because the crystal structure does not represent the solution structure of hemoglobin very well (Cammarata et al., 2008). It is obvious that the MARTINI coarse-grained calculations could be used for any refinement algorithm to improve the agreement, but that the amino acid approach would not contain enough structural detail to achieve this. Conversely, such refinement schemes are very likely to benefit from the reduced computational cost of the MARTINI representation relative to the atomistic scattering model.

The calculations shown in Fig. 4 include the displacedsolvent term, but any excess electron density of the solvation layer was neglected. This is reasonable when considering difference scattering, as errors cancel to some degree when taking differences. The data in Fig. 5 demonstrate this. The difference scattering for three systems is shown: sperm whale myoglobin (deoxy and carbonmonoxy state), human hemoglobin (deoxy and carbonmonoxy state) and deinococcus radiodurans phytochrome ( $\mathrm{Pr}$ and $\mathrm{Pfr}$ state). These systems were selected since they cover a wide range of magnitudes in conformational change as shown by the root-mean-square deviations of the respective structure pairs ( $c f$. Fig. 5). The two solution difference scattering curves for each test system are computed by (i) considering the atomic scattering and the displaced solvent [as formulated in equation (4)], and (ii) additionally including the scattering due to the solvation layer. The data were calculated using CRYSOL (Svergun et al.,

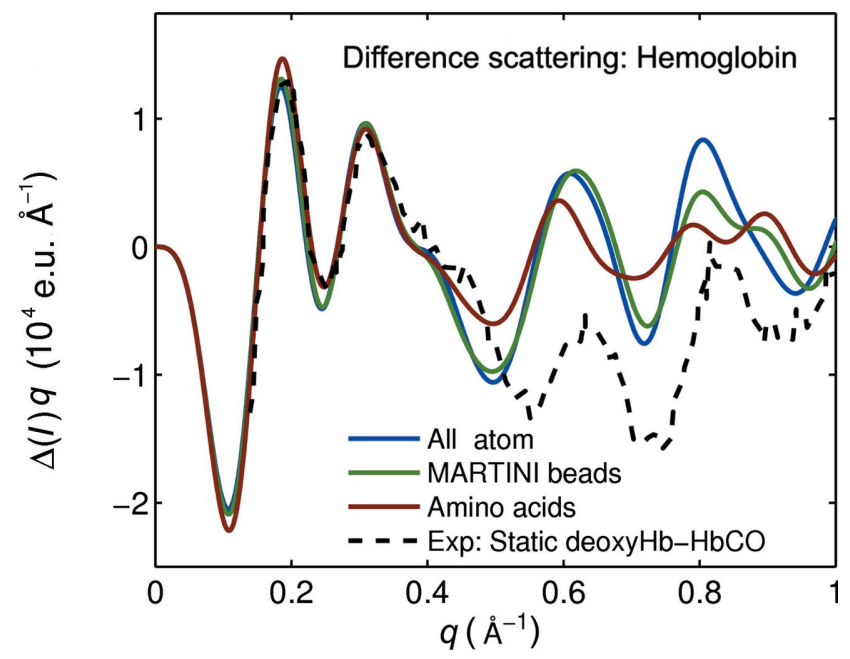

Figure 4

Calculated solution difference scattering between human deoxy and carbonmonoxy hemoglobin crystal models (2hhb-1bbb), compared with experimental solution data from Cammarata et al. (2008). The cofactors were not taken into account in the calculation. The experimental curve has been scaled to the calculated data at $q=0.18 \AA^{-1}$. 
1995), with its highest resolution $(L=50)$ and default parameters for the solvation shell and displaced solvent (Svergun et al., 1995). The program approximates the solvation layer effect by assuming a uniform electron distribution around the protein that differs from bulk water by $+10 \%$, which is known to be inaccurate in the high- $q$ region (Park et al., 2009). However, the simple solvation layer treatment in CRYSOL can be used as a prototype to estimate the effect of a solvation layer model on the calculation of difference scattering. It is evident that the calculation with displaced solvent is in good agreement with the one that additionally models the solvation layer scattering for all three test systems.

\subsection{Reliability of absolute $X$-ray scattering calculated from coarse-grained protein structures}

We now turn our attention to assessing the accuracy of the calculations of absolute X-ray scattering from coarse-grained structures. Fig. 6 shows the effect of coarse-graining on X-ray scattering calculations for a hen egg-white lysozyme [PDB code 6lyz (Diamond, 1974); Fig. 6(a)] and human carbon-

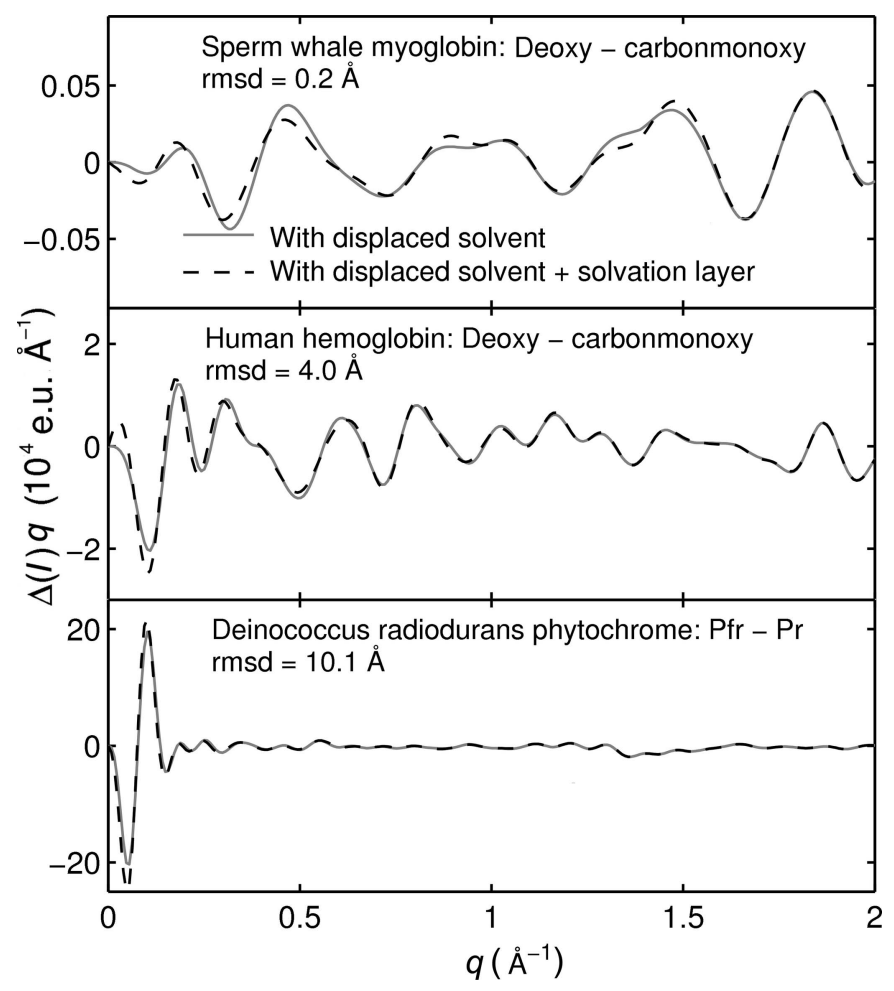

Figure 5

Solution difference scattering of three model systems with different magnitudes of conformational changes calculated with CRYSOL (Svergun et al., 1995). The root-mean-square deviations (rmsd) for the respective conformers are shown in the panels. To evaluate the effect of displaced solvent and solvation layer scattering, two difference curves are shown: (i) atomic X-ray scattering with displaced solvent and (ii) atomic $\mathrm{X}$-ray scattering with displaced solvent and solvation layer. The following structures were used (PDB codes in brackets): sperm whale deoxy myoglobin $(2 \mathrm{~g} 0 \mathrm{v}$, second state; Aranda et al., 2006), sperm whale carbonmonoxy myoglobin (2g0r; Aranda et al., 2006), human deoxy hemoglobin (2hhb) and human carbonmonoxy hemoglobin (1bbb); these structures were used without cofactors. The deinococcus radiodurans phytochrome solution structures were taken from Takala et al. (2014). monoxy hemoglobin [PDB code 2hhb, Fig. 6(a)]. These structures are not part of the protein structure library used to derive the coarse-grained form factors. For both structures, the agreement between the all-atom calculation and the coarsegrained calculations are good for low $q$ and start to become worse at higher $q$. As expected, coarse-graining according to the MARTINI scheme agrees with the all-atom calculation to higher $q$ than the amino acid approach. To quantify this agreement we use the average relative squared error in the range from 0 to $q(N)$ with $N$ being the number of data points in the respective range:

$$
\text { error }=\frac{1}{N} \sum_{q=0}^{q(N)} \frac{\left(S_{\mathrm{aa}}-S_{\mathrm{cg}}\right)^{2}}{S_{\mathrm{aa}}^{2}}
$$

The dotted lines in Fig. 6 mark the maximum $q$ value for the coarse-grained calculations $\left(q_{\text {threshold }}\right)$, for which the error [equation (12)] is smaller than $0.2 \%$. The error limit of $0.2 \%$ is arbitrary but was chosen with respect to the absolute scattering curves of hen egg-white lysozyme (Fig. 6a). For hen eggwhite lysozyme and human carbonmonoxy hemoglobin, the $q_{\text {threshold }}$ values are 0.31 and $0.25 \AA^{-1}$, respectively, for the

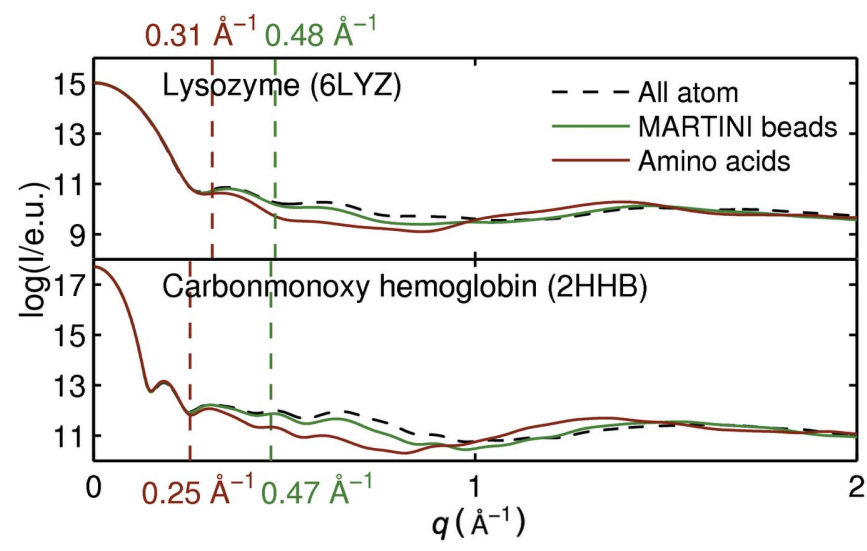

(a)

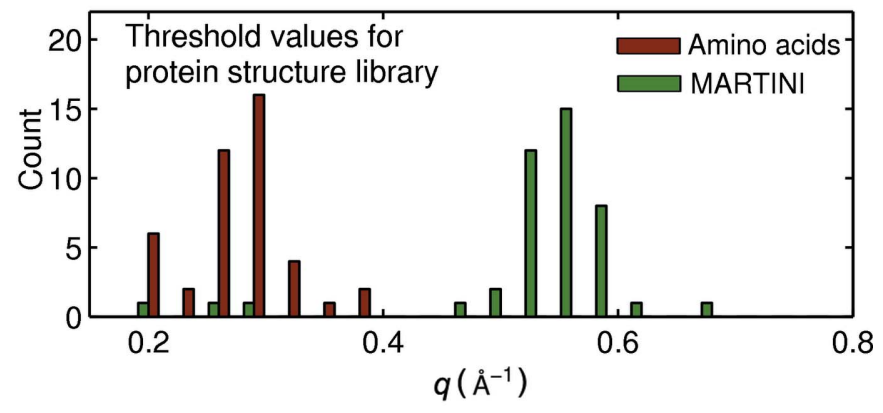

(b)

Figure 6

(a) Determination of $q_{\text {threshold }}$ (see text for details) for reliable coarsegrained calculation of protein X-ray scattering for hen egg-white lysozyme (PDB code 6lyz) and carbonmonoxy hemoglobin (PDB code $2 \mathrm{hhb}$ ). The cofactors were removed before evaluating the scattering. (b) For each structure of the protein structure library, the maximum $q$ value with an error lower than $0.2 \%$ [according to equation (12)] was determined. The histogram illustrates the distribution of these $q_{\text {threshold }}$ values for both coarse-grained methods (amino acid and MARTINI). 
amino acid approach and 0.48 and $0.47 \AA^{-1}$, respectively, for the MARTINI bead approach.

The $q_{\text {threshold }}$ [equation (12)] values for the amino acid and the MARTINI approach for all proteins in the library are depicted as a histogram in Fig. 6(b). It is evident that the MARTINI coarse-grained calculations provide a wider $q$ range (on average $0.53 \AA^{-1}$ ) compared with the amino acid bead approach (on average $0.27 \AA^{-1}$ ). This shows that the use of MARTINI beads significantly extends the range in which scattering can be reliably calculated compared with amino acid coarse-graining.

We note that the results presented in Fig. 6 do not include a model of the solvation layer around the protein. A number of sophisticated methods to incorporate this are already available, and this is critical for comparison with absolute experimental SAXS/WAXS data (Grishaev et al., 2010; Park et al., 2009; Bardhan et al., 2009). However, the underlying physics, which is that a coarser representation of structure leads to a loss in resolution, is well captured in the model that was used to compute the data shown in Fig. 6.

\section{Discussion}

The increasingly popular method of time-resolved WAXS requires advanced computational structural refinement schemes. In this paper, we have shown that coarse-graining the structures leads to a loss of accuracy at wide angles. Thus, when choosing the coarseness of the structural model, computational cost should be carefully balanced against the accuracy needed, a decision which critically depends on the $q$ range of interest. For the case of human hemoglobin presented above, high-resolution crystal models deviate from experimental solution data for $q>0.4 \AA^{-1}$. Thus a MARTINI representation and scattering model would be suitable for interpreting the available difference data up to $q=0.75 \AA^{-1}$.

To the best of our knowledge, there are three refinement schemes for time-resolved X-ray scattering experiments of proteins. Ahn et al. (2009) have successfully applied a biased molecular dynamics simulation to time-resolved X-ray scattering data, Andersson et al. (2009) and Ahn et al. (2009) moved rigid bodies, and Kim, Lee et al. (2012) used ab initio determination of the three-dimensional structure. For these three approaches, a large number of X-ray scattering calculations had to be performed and this was the limiting factor in the studies. A treatment of larger proteins becomes prohibitively expensive. The MARTINI coarse-grained calculations offer a good compromise between accuracy in the X-ray scattering calculation and computational speed. The computation of X-ray scattering from coarse-grained protein structures on the MARTINI level is 50 times faster than the corresponding all-atom calculation (around 7-8 atoms go into the average MARTINI bead, $7^{2}=49$ ). This speed-up could break new ground for applying difference scattering-assisted structural refinement to larger proteins. ${ }^{1}$

\footnotetext{
$\overline{{ }^{1}}$ The form factors are available from the IUCr electronic archives (Reference: AJ5230).
}

When many trial structures are to be evaluated, using computationally demanding state-of-the-art methods to account for solvation effects is not feasible. We show here that less sophisticated solvation treatment can be used to reliably model difference WAXS. This is rationalized by the fact that the shape of the solvent shell does not change very much between different protein conformations, and that its contribution to the scattering simply cancels out in the difference scattering. Another advantage of using difference scattering as an experimental observable is that it is free of experimental artifacts stemming from incorrect subtraction of scattering from the buffer and the capillary. This means that the discrepancy between calculation and experiment significantly diminishes compared with standard SAXS/WAXS.

\section{Conclusion}

The speed of computations of X-ray scattering from (bio)molecules can be controlled by coarse-graining the underlying structures. Our results provide the basis for matching the level of coarse-graining with the required resolution. When the beads contain entire amino acids and for the finer MARTINI scheme, we estimate reliability $q$ ranges of 0 $0.3 \AA^{-1}$ and $0-0.5 \AA^{-1}$, respectively. The MARTINI coarsegrained model thus covers the $q$ range available in standard SAXS experiments and is 50 times faster than the all-atom calculation. When difference X-ray scattering is analyzed, for example, in time-resolved SAXS/WAXS, the reliability $q$ range is significantly extended to $0.75 \AA^{-1}$, which we showed for the model system human hemoglobin. We anticipate that the increased efficiency in computation of protein X-ray scattering will enable more comprehensive structural analyses in the growing field of time-resolved difference X-ray scattering of proteins.

The authors thank Professor Sichun Yang for fruitful discussions. Funding by the ERC grant 'StructDyn' and the FFL4 program of the Foundation of Strategic Research, Sweden, is also acknowledged.

\section{References}

Ahn, S., Kim, K., Kim, Y., Kim, J. \& Ihee, H. (2009). J. Phys. Chem. B, 113, 13131-13133.

Andersson, M., Malmerberg, E., Westenhoff, S., Katona, G., Cammarata, M., Wöhri, A. B., Johansson, L. C., Ewald, F., Eklund, M., Wulff, M., Davidsson, J. \& Neutze, R. (2009). Structure, 17, 1265-1275.

Aranda, R., Levin, E. J., Schotte, F., Anfinrud, P. A. \& Phillips, G. N. (2006). Acta Cryst. D62, 776-783.

Bardhan, J., Park, S. \& Makowski, L. (2009). J. Appl. Cryst. 42, 932943.

Bogaart, G. van den, Meyenberg, K., Risselada, H. J., Amin, H., Willig, K. I., Hubrich, B. E., Dier, M., Hell, S. W., Grubmüller, H., Diederichsen, U. \& Jahn, R. (2011). Nature, 479, 552-555.

Cammarata, M., Levantino, M., Schotte, F., Anfinrud, P., Ewald, F., Choi, J., Cupane, A., Wulff, M. \& Ihee, H. (2008). Nat. Methods, 5, 881-886.

Cho, H., Dashdorj, N., Schotte, F., Graber, T., Henning, R. \& Anfinrud, P. (2010). Proc. Natl Acad. Sci. USA, 107, 7281-7286. 
Daily, M. D., Makowski, L., Phillips, G. N. Jr \& Cui, Q. (2012). Chem. Phys. 396, 84-91.

Diamond, R. (1974). J. Mol. Biol. 82, 371-391.

Fermi, G., Perutz, M. F., Shaanan, B. \& Fourme, R. (1984). J. Mol. Biol. 175, 159-174.

Fraser, R. D. B., MacRae, T. P. \& Suzuki, E. (1978). J. Appl. Cryst. 11, 693-694.

Grishaev, A., Guo, L., Irving, T. \& Bax, A. (2010). J. Am. Chem. Soc. 132, 15484-15486.

Harker, D. (1953). Acta Cryst. 6, 731-736.

Hess, B., Kutzner, C., van der Spoel, D. \& Lindahl, E. (2008). J. Chem. Theory Comput. 4, 435-447.

Ibrahimkutty, S., Kim, J., Cammarata, M., Ewald, F., Choi, J., Ihee, H. \& Plech, A. (2011). ACS Nano, 5, 3788-3794.

Ihee, H., Wulff, M., Kim, J. \& Adachi, S. (2010). Int. Rev. Phys. Chem. 29, 453-520.

Jong, D. H. de, Singh, G., Bennett, W. F. D., Arnarez, C., Wassenaar, T. A., Schäfer, L. V., Periole, X., Tieleman, D. P. \& Marrink, S. J. (2013). J. Chem. Theory Comput. 9, 687-697.

Kim, K. H., Muniyappan, S., Oang, K. Y., Kim, J. G., Nozawa, S., Sato, T., Koshihara, S., Henning, R., Kosheleva, I., Ki, H., Kim, Y., Kim, T. W., Kim, J., Adachi, S. \& Ihee, H. (2012). J. Am. Chem. Soc. 134, 7001-7008.

Kim, T. W., Lee, J. H., Choi, J., Kim, K. H., van Wilderen, L., Guerin, L., Kim, Y., Jung, Y. O., Yang, C., Kim, J., Wulff, M., van Thor, J. \& Ihee, H. (2012). J. Am. Chem. Soc. 134, 3145-3153.

Koch, M. H., Vachette, P. \& Svergun, D. I. (2003). Q. Rev. Biophys. 36, 147-227.

Konarev, P. V., Petoukhov, M. V., Volkov, V. V. \& Svergun, D. I. (2006). J. Appl. Cryst. 39, 277-286.

Liu, H., Hexemer, A. \& Zwart, P. H. (2012). J. Appl. Cryst. 45, 587593.

López, C., Rzepiela, A., de Vries, A., Dijkhuizen, L., Hünenberger, P. \& Marrink, S. (2009). J. Chem. Theory Comput. 5, 3195-3210.

Louhivuori, M., Risselada, H. J., van der Giessen, E. \& Marrink, S. J. (2010). Proc. Natl Acad. Sci. USA, 107, 19856-19860.

Makowski, L. (2010). J. Struct. Funct. Genomics, 11, 9-19.

Malmerberg, E., Omran, Z., Hub, J. S., Li, X., Katona, G., Westenhoff, S., Johansson, L. C., Andersson, M., Cammarata, M., Wulff, M., van der Spoel, D., Davidsson, J., Specht, A. \& Neutze, R. (2011). Biophys. J. 101, 1345-1353.
Marrink, S. J., Risselada, H. J., Yefimov, S., Tieleman, D. P. \& de Vries, A. H. (2007). J. Phys. Chem. B, 111, 7812-7824.

Marrink, S. J. \& Tieleman, D. P. (2013). Chem. Soc. Rev. 42, 68016822.

Monticelli, L., Kandasamy, S., Periole, X., Larson, R., Tieleman, D. \& Marrink, S. (2008). J. Chem. Theory Comput. 4, 819-834.

Oberg, K., Ruysschaert, J. \& Goormaghtigh, E. (2003). Protein Sci. 12, 2015-2031.

Park, S., Bardhan, J., Roux, B. \& Makowski, L. (2009). J. Chem. Phys. 130, 134114.

Petoukhov, M. V., Franke, D., Shkumatov, A. V., Tria, G., Kikhney, A. G., Gajda, M., Gorba, C., Mertens, H. D. T., Konarev, P. V. \& Svergun, D. I. (2012). J. Appl. Cryst. 45, 342-350.

Petoukhov, M. V. \& Svergun, D. I. (2007). Curr. Opin. Struct. Biol. 17, 562-571.

Schäfer, L. V., de Jong, D. H., Holt, A., Rzepiela, A. J., de Vries, A. H., Poolman, B., Killian, J. A. \& Marrink, S. J. (2011). Proc. Natl Acad. Sci. USA, 108, 1343-1348.

Silva, M. M., Rogers, P. H. \& Arnone, A. (1992). J. Biol. Chem. 267, 17248-17256.

Spilotros, A., Levantino, M., Schiro, G., Cammarata, M., Wulff, M. \& Cupane, A. (2012). Soft Matter, 8, 6434-6437.

Stovgaard, K., Andreetta, C., Ferkinghoff-Borg, J. \& Hamelryck, T. (2010). BMC Bioinformatics, 11, 429.

Svergun, D., Barberato, C. \& Koch, M. H. J. (1995). J. Appl. Cryst. 28, 768-773.

Svergun, D. I. \& Koch, M. H. (2003). Rep. Progr. Phys. 66, 1735.

Takala, H., Björling, A., Berntsson, O., Lehtivuori, H., Niebling, S., Hoernke, M., Kosheleva, I., Henning, R., Menzel, A., Ihalainen, J. \& Westenhoff, S. (2014). Nature, 509, 245-249.

Virtanen, J. J., Makowski, L., Sosnick, T. R. \& Freed, K. F. (2011). Biophys. J. 101, 2061-2069.

Warren, B. E. (1990). X-ray Diffraction. New York: Dover Publications Inc.

Westenhoff, S., Nazarenko, E., Malmerberg, E., Davidsson, J., Katona, G. \& Neutze, R. (2010). Acta Cryst. A66, 207-219.

Yang, S., Park, S., Makowski, L. \& Roux, B. (2009). Biophys. J. 96, 4449-4463.

Yesylevskyy, S., Schäfer, L., Sengupta, D. \& Marrink, S. (2010). PLoS Comput. Biol. 6, e1000810.

Zheng, W. \& Tekpinar, M. (2011). Biophys. J. 101, 2981-2991. 